\title{
Review
}

\section{Crimean-Congo hemorrhagic fever: Current and future prospects of vaccines and therapies}

\author{
Maryam Keshtkar-Jahromi ${ }^{a}$, Jens H. Kuhn ${ }^{b}$, Iva Christova ${ }^{c}$, Steven B. Bradfute ${ }^{d}$, \\ Peter B. Jahrling ${ }^{\mathrm{b}}$, Sina Bavari ${ }^{\mathrm{d}, *}$ \\ a Union Memorial Hospital, University of Maryland, Baltimore, MD, USA \\ ${ }^{\mathrm{b}}$ Integrated Research Facility at Fort Detrick, National Institute of Allergy and Infectious Diseases, National Institutes of Health, Fort Detrick, Frederick, MD, USA \\ ${ }^{\mathrm{c}}$ National Centre of Infectious and Parasitic Diseases, Sofia, Bulgaria \\ ${ }^{\mathrm{d}}$ United States Army Medical Research Institute of Infectious Diseases, Fort Detrick, Frederick, MD, USA
}

\section{A R T I C L E I N F O}

\section{Article history:}

Received 24 November 2010

Received in revised form 4 February 2011

Accepted 21 February 2011

Available online 6 March 2011

\section{Keywords:}

Crimean-Congo hemorrhagic fever

Bunyavirus

Nairovirus

Priority pathogen

Ribavirin

Antiviral therapy

Viral hemorrhagic fever

\begin{abstract}
A B S T R A C T
Crimean-Congo hemorrhagic fever (CCHF) is a tick-borne disease caused by CCHF virus (CCHFV), a nairovirus in the family Bunyaviridae. CCHF occurs sporadically in a number of countries in Asia, the Middle East, southeastern Europe and Africa. Patients may develop subclinical to severe hemorrhagic disease, with fatal outcomes in a substantial percentage of cases. Transmission usually occurs through contact with viremic livestock or patients or bites by infected ticks. The number of reported cases has increased in recent years, possibly due to global climatic change and human perturbations of biocenoses that may have led to the migration of tick vectors. There is currently no FDA-approved vaccine or specific antiviral therapy for CCHF. The classification of CCHFV as a WHO Risk Group IV pathogen and the lack of suitable animal models has caused progress in developing new prophylactic and therapeutic measures to be slow. Ribavirin is active against CCHFV in vitro, but its efficacy for human therapy has not been definitively demonstrated by clinical studies. CCHF-immunoglobulin is also in use, but without clear evidence of efficacy. In this article, we review the development of prophylaxis and therapy for CCHF and discuss future prospects for vaccine and drug development.
\end{abstract}

(C) 2011 Published by Elsevier B.V.

\section{Contents}

1. Introduction

2. Vaccines

3. Animal models.

4. Treatment

4.1. Supportive therapy

4.2. Ribavirin...

4.2.1. Activity in vitro and in laboratory animals

4.2.2. Ribavirin efficacy in CCHF patients

4.2.3. Ribavirin therapy: conclusions.

Abbreviations: ALT, alanine aminotransferase; CCHF, Crimean-Congo hemorrhagic fever; CCHFV, Crimean-Congo hemorrhagic fever virus; CCL, chemokine (C-C motif)

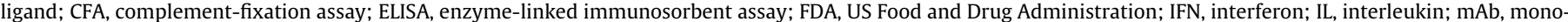

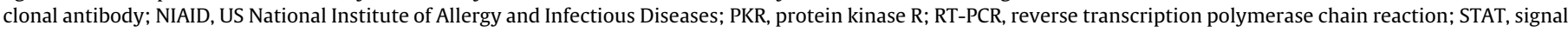
transducer and activator of transcription; TNF, tumor necrosis factor; VLP, virus-like particle; WHO, World Health Organization.

is Disclaimer: This work was partially supported by the Defense Threat Reduction Agency, Transformational Medical Technologies project number 0048-09-RD-T (to SB).

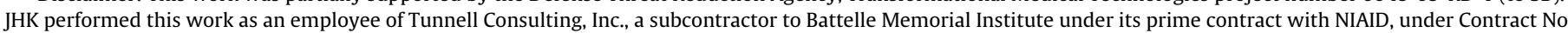
HHSN272200200016I. Opinions, interpretations, conclusions, and recommendations are those of the authors and are not necessarily endorsed by the US Army, the US Department of Health and Human Services or of the institutions and companies affiliated with the authors.

* Corresponding author at: United States Army Medical Research Institute of Infectious Diseases, 1425 Porter Street, Fort Detrick, Frederick, MD 21702, USA. Tel: +1 301619 4246; fax: +1 5417543545 .

E-mail address: sina.bavari@us.army.mil (S. Bavari). 


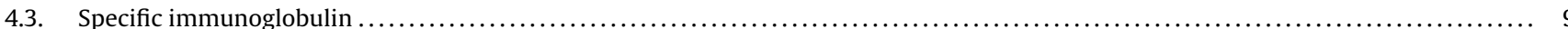

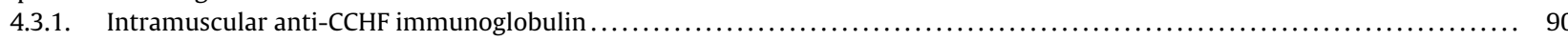

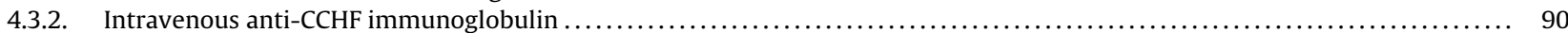

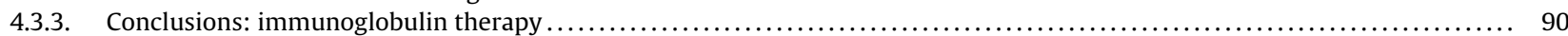

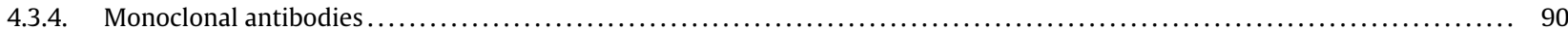

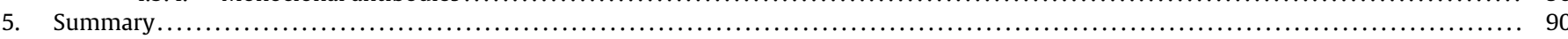

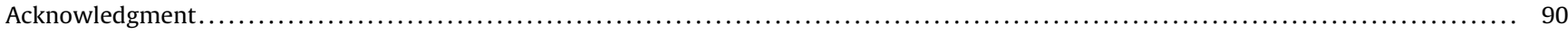

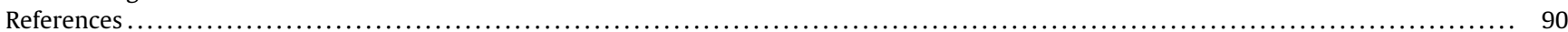

\section{Introduction}

Crimean-Congo hemorrhagic fever virus (CCHFV), a member of the genus Nairovirus in the family Bunyaviridae, causes what today is referred to as Crimean-Congo hemorrhagic fever (CCHF) (Haenni et al., 2005). The disease was first recognized towards the end of World War II, when some 200 Soviet military personnel and peasants fell ill in devastated western Crimea (Chumakov, 1945). However, in 1956, a disease very similar to CCHF befell a child in the Belgian Congo (today the Democratic Republic of the Congo). Between 1956 and 1965, this virus was isolated (Simpson et al., $1965,1967)$ and demonstrated to be identical to that causing the Soviet cases (Casals, 1969; Woodall, 2007).

CCHF usually develops after humans have been bitten by ixodid ticks or have been in contact with infected animals (most often livestock) or humans, or their tissues, excreta or secreta (Ergonul and Whitehouse, 2007). The disease occurs in four phases, designated the incubation, prehemorrhagic, and hemorrhagic periods and convalescence (Ergonul, 2007). After an incubation period of 1-9 days, patients present with a nonspecific influenza-like syndrome that typically lasts less than one week (Mardani and Keshtkar-Jahromi, 2007). In some cases, the hemorrhagic period develops rapidly, beginning between the third and fifth day of illness. Circulatory shock and disseminated intravascular coagulation may occur in severe cases (Ergonul, 2007; Mardani et al., 2003; Swanepoel et al., 1989). The clinical symptoms of children and adolescents do not differ from those of adults. In Iran, children seem to be more vulnerable to severe disease than adults, whereas in Turkey lethal cases of CCHF have rarely been observed among children (Sharifi-Mood et al., 2008; Tezer et al., 2010).

The diagnosis of CCHF has generally been based on the detection of anti-CCHFV IgM or a four-fold increase in antibody titer from paired serum samples. However, patients who die during the first four days of disease do not develop antibodies and therefore elude serologic diagnosis (Ergonul, 2006; Vorou et al., 2007). In that case, RT-PCR is recommended, as this test can be highly specific, sensitive, and rapid (Ergonul, 2006). Together, this means that depending on the familiarity of clinicians with the disease, the point in the course of illness and the severity of the patient's condition, CCHF may be difficult differentiate from febrile illnesses.

Today, it is clear that CCHFV is one of the most widely spread tick-borne viruses of medical importance. It is endemic to Asia, Eastern Europe, the Middle East, and central and southern Africa, and it causes human disease in increasing numbers per year in many countries, including Turkey and possibly the successor nations of the Soviet Union (Ergonul and Whitehouse, 2007). Nevertheless, CCHF is a sporadic, uncommon illness even in those countries with a rather rich history of cases (Kazakhstan, Russia, South Africa). Few physicians have had hands-on experience with CCHF patients, medical response times tend to be prolonged because of delayed diagnosis, and the gathering of epidemiological data is hindered by the rural infrastructure in outbreak areas.
WHO classifies CCHFV in WHO Risk Group IV, meaning that maximum biocontainment facilities are recommended for any research that uses infectious virus. In the USA, CCHFV is classified as a Select Agent, a Risk Group 4 Pathogen, and a NIAID Category C Priority Pathogen because of the absence of efficacious prophylactic or treatment regimens. While the classification as a Priority Pathogen has released funds to study CCHFV, the requirement to work with it under Biosafety Level 4 conditions has limited the number of researchers with access to the pathogen. CCHFV is considered a potential biological weapon (Bronze et al., 2002). However, the same technical challenges that hinder progress in the molecular characterization of the virus and the development of effective prophylaxes for or vaccines against it most likely preclude its development as a mass-casualty weapon (Borio et al., 2002). On the other hand, CCHF is of interest to military forces, because it is endemic in many areas where soldiers may be deployed, either for conflicts or humanitarian aid. The fatal infection of an American soldier in southern Afghanistan in 2009 was a grave reminder of the risk posed by this disease (Carter, 2009).

\section{Vaccines}

Initial attempts to develop vaccines date back to the 1960s, when Soviet scientists advocated the immunization of local populations because of CCHFV endemicity. Nonspecific preventive measures such as tick eradication had proved to be expensive, inefficient and in many instances impractical (Badalov et al., 1969). Researchers of the Soviet Institute of Poliomyelitis and Viral Encephalitides developed an experimental CCHF vaccine based on brain tissue from infected newborn laboratory mice and rats. Brain tissue suspensions were inactivated by formaldehyde and heat treatment to obtain safe, noninfectious preparations. The efficacy of the vaccine was tested using the complement fixation ( $\mathrm{CF}$ ) technique and titrated against immune sera collected from CCHF convalescent patients or experimentally infected animals (Tkachenko et al., 1970). The immunogenicity of the vaccine was evaluated by serial intraperitoneal injections into newborn rats at 7-day intervals. Antibodies of all classes could be detected one week after the first booster injection (Tkachenko et al., 1970). The vaccine did not have adverse affects on the limited number of humans who volunteered to be vaccinated.

In 1970, this inactivated vaccine was approved by the Soviet Ministry of Health for CCHF prophylaxis (Tkachenko et al., 1970). In the same year, serum samples were collected from some 2000 healthy people before vaccination, two weeks after a 2nd injection and two weeks, one month, three to four months, and six months after a 3rd injection. The sera were tested for the presence of antiCCHFV antibodies using CF and agar gel diffusion and precipitation techniques. Low levels of antibodies $(\leq 5.7 \%$ one month after the third injection) were detected, and $42 \%$ of samples tested positive using both tests. Neutralizing antibodies developed within 1-4 weeks after the 3rd injection, but titers decreased 3-6 months later (Tkachenko et al., 1971). Approximately 1500 people vaccinated 
in 1970 were revaccinated once in 1971, resulting in an increased seroresponse at one month and six months, which correlated with the number of original injections (Tkachenko et al., 1971; Vasilenko, 1973). Unfortunately, no data regarding the protective efficacy of the vaccine have been published.

In 1974, the Soviet vaccine was licensed in Bulgaria and used in CCHFV-endemic areas of the country for military personnel and medical and agricultural workers over 16 years of age. Recently published data from the Bulgarian Ministry of Health suggest a four-fold reduction in the number of reported CCHF cases over a 22-year time period (1953-1974: 1105 cases; 1975-1996: 279 cases) (Christova et al., 2010). Since 1997, less than 20 cases have been reported annually to the ministry, though more have probably occurred. Notably, none of the vaccinated military personnel have contracted $\mathrm{CCHF}$, and none of the vaccinated laboratory personnel working with CCHF virus became infected even after occasional exposures by needle pricking. Although there are no published data regarding the total number of vaccinated civilians who have developed CCHF, these data nevertheless suggest that the vaccine is efficacious (Christova et al., 2010). Of course, it is also possible that case reporting has changed since vaccination was instituted, or that populations have changed their behavior and reduced their tick exposure. It is also possible that the epidemiology of CCHF and the ecology of CCHFV have changed over the years in Bulgaria without any intentional intervention. Regrettably, there are no data regarding the incidence of CCHF in the same time frame in neighboring countries, preventing further analysis of vaccine efficacy.

In a recent Bulgarian study, significant antibody responses were observed in vaccinated volunteers who were regularly immunized at 2-years intervals for the last 15-20 years. Antibody levels were measured before each new booster dose and 2, 3 and 4 weeks later. Using CF assays, titers of $1: 2$ to $1: 16$ were detected before re-vaccination, followed by a 2-4-fold increase $2-3$ weeks after revaccination. Consistent, high antibody titers were detected by ELISA before and after revaccination. The antibody response before the $3^{\text {rd }}$ injection was either zero, borderline positive, or weakly positive, but a significant increase was detected by both CFA and ELISA thereafter (I. Christova, unpublished data).

As described by the manufacturer, the Bulgarian vaccine consists of Active substance: inactivated CCHFV antigen; and Auxiliary substances: aluminum hydroxide, thiomersal, sodium hydrogen carbonate, sodium chloride, phenol red as an indicator, tobramycin, and water (Anon., 2008). A dose of $1 \mathrm{~mL}$ subcutaneously is followed by a second injection 30-45 days later, re-immunization one year later and then every five years. The vaccine is designed for persons over 16 years of age. For persons who have been treated with CCHF-specific immunoglobulin, 30 days should pass before immunization. Corticosteroid usage or immunosuppressive treatment may render vaccination unsuccessful. Vaccination may be accompanied with local reactions or fever. It is not deemed necessary for persons who have recovered from CCHF. This vaccine requires the use of maximum containment facilities to generate virions for inactivation. There is also concern about using vaccines grown in mouse brains due to possible autoimmune and allergic responses induced by myelin basic protein (Hemachudha et al., 1987; Jelinek, 2008). Additional vaccine platforms should therefore be considered.

Modern vaccine development foresees the establishment of DNA vaccines, recombinant viral protein-based vaccines, and virus-like particle vaccines. However, research has been severely hindered by the absence of CCHF animal models. For instance, American scientists have developed a DNA vaccine containing the CCHF genome $M$ segment and have shown that it elicited neutralizing antibodies in mice, as well as antibodies that immunoprecipitated M-segment expression products (Spik et al., 2006). However, they were unable to evaluate the vaccine's protective efficacy.

\section{Animal models}

Animal models of CCHF have until recently been limited to intracranial or intraperitoneal infection of newborn mice or rats with the suckling mouse-passaged IbAr 10200 or Hodzha CCHFV strains (Smirnova, 1979; Smirnova et al., 1973; Tignor and Hanham, 1993). Although the use of these animals has generated some interesting results, newborn mice are unusually susceptible to a wide array of pathogens, and their usefulness as disease models is questionable. However, CCHFV-infected adult mice, rats, guinea pigs, hamsters, rabbits, sheep, calves, donkeys, nonhuman primates, and other adult animals exhibit low to undetectable viremia and clear the infection without overt signs of illness (Fagbami et al., 1975; Shepherd et al., 1989; Smirnova, 1979).

Recently, two models of lethal CCHFV infection have been reported in adult mice with defective interferon responses (Bente et al., 2010; Bereczky et al., 2010). In the first model, knockout mice lacking the signal transducer and activator of transcription-1 (STAT-1) protein died 3-5 days after infection with low doses of the mouse-passaged CCHFV strain IbAr 10200, and developed thrombocytopenia and leukopenia, as well as increased serum ALT levels, which are also found in lethal human infections, supporting the validity of STAT-1 KO mice as a model of human CCHF (Bente et al., 2010; Joubert et al., 1985; Swanepoel et al., 1989). The animals also developed characteristic histopathologic lesions, including necrosis in the livers and lymphocyte depletion in the spleens, but interstitial pneumonia and intestinal hemorrhage, found in lethal human infections, were not apparent. Analysis of immune cells suggested activation of macrophages, dendritic cells, and NK cells in lethal infection, although there was no comparison with these cells in infected wild-type mice. Infected mice also had elevated serum IL-6, IL-10, and TNF- $\alpha$ concentrations, which correlate with findings in some human cases (Ergonul et al., 2006; Papa et al., 2006). The model was also used to demonstrate that ribavirin therapy could prevent lethal infection.

The second model employs knockout mice lacking the cellsurface IFN- $\alpha / \beta$ receptor (IFNAR ${ }^{-/-}$), in which infection with CCHFV strain IbAr 2000 was lethal within 2-4 days (Bereczky et al., 2010). Viral replication was highest in the liver and spleen, and up to 10,000 -fold higher in IFNAR $^{-1-}$ mice than in immunocompetent mice. Enlarged, hemorrhagic livers were found in IFNAR ${ }^{-1-}$ mice infected with lower $\left(10^{5}, 10^{3}\right.$ and $10^{1}$ focus-forming units $)$, but not higher $\left(10^{6}\right.$ focus-forming units), doses of virus.

Both mouse models can potentially serve as platforms for studies aiming to analyze the pathogenesis and treatment of CCHF, such as inflammatory cytokine production or the effectiveness of immune serum therapy. Vaccine studies, which were not feasible in newborn mice, might also be applicable using these platforms. Whether or not CCHFV can be adapted for studies in other animals, it is possible that suppression of type I interferon responses would allow for lethal infection. The use of humanized mice may also be useful for further model development (Brehm et al., 2010). It is now necessary to focus on the further characterization of these new models, since at least in the USA, two well-characterized animal models reflecting human disease parameters should ideally be available for the approval of any drug or vaccine for human use, under the FDA “Animal Rule."

Although it appears that mouse models can be developed further to satisfy the stringent FDA requirements for drug approval, the current lack of a CCHF model in higher mammals, such as nonhuman primates, raises the question how new drugs and vaccines could be tested in human populations without the use of the "Animal Rule." Could the evaluation of new treatments be ethically justified within international collaborations in countries with less stringent rules for drug testing? If so, which countries would participate in such trials, and under what circumstances? Is CCHF so "exotic" a disease 
that the development of new therapies can be postponed until a satisfactory animal model has been found? The initiation of such discussions is an urgent matter that should not be further delayed.

\section{Treatment}

Most CCHF patients receive only supportive therapy. Although ribavirin has been employed for over 25 years for the prophylaxis and therapy of CCHF, its efficacy remains controversial. Below we summarize all major published reports in the English-language literature of the clinical use of ribavirin for CCHF, and then comment on the need for further investigation of its therapeutic benefit. We then briefly review experience with the use of specific immunoglobulin for the treatment of CCHF in the former Soviet Union and Bulgaria and the potential development of monoclonal antibody therapies.

\subsection{Supportive therapy}

CCHF patients must be monitored closely for effective support. Measurement of the complete blood count, serum electrolyte levels, and coagulation indices is crucial, and transfusions with blood products must be carefully considered, based on individual deficits. Medical staff should be aware of the possibility of lifethreatening hemorrhages; non-steroidal anti-inflammatory drugs are therefore contraindicated, and intramuscular injections should be avoided (Ergonul, 2006). In Turkey, two cases of CCHF complicated by hypothermia and severe hemorrhagic manifestations were recently treated successfully with only fresh frozen plasma, crystalloid, and colloid infusions, indicating that supportive measures may be sufficient even in severe cases (Yilmaz et al., 2009).

\subsection{Ribavirin}

\subsubsection{Activity in vitro and in laboratory animals}

Ribavirin is a purine nucleoside analogue with broad-spectrum antiviral activity. Indirect (inosine monophosphate dehydrogenase inhibition, immunomodulatory effects) and direct mechanisms (interference with mRNA capping, polymerase inhibition, lethal mutagenesis) have been proposed to explain its antiviral activity (Graci and Cameron, 2006). The efficacy of ribavirin against CCHFV was first described in vitro in 1989, when the drug markedly reduced viral yields of variants from Europe, Asia, and Africa (Watts et al., 1989). Although some isolates appeared to be more sensitive than others, CCHFV proved in general to be more sensitive to ribavirin than a bunyaviral relative, Rift Valley fever virus (genus Phlebovirus). A recent study confirmed that ribavirin inhibited CCHFV replication according to plaque-reduction assays, and no significant differences in drug sensitivity of different viral isolates were observed (Paragas et al., 2004). In a study in suckling mice using the mouse-passaged IbAr 10200 CCHF strain, ribavirin significantly reduced lethality, increased the mean time to death, reduced viral replication in the liver and prevented infection of brain and heart tissues (Tignor and Hanham, 1993). The protective activity of ribavirin has also been demonstrated in CCHFV-infected STAT-1 knockout mice (Bente et al., 2010).

\subsubsection{Ribavirin efficacy in CCHF patients}

4.2.2.1. Observational studies. The first reported clinical use was during a nosocomial outbreak in a South African hospital in 1985 (Table 1) (van de Wal et al., 1985). Six of 9 healthcare workers with penetrating injuries from CCHFV-contaminated needles were treated with intravenous ribavirin and interferon- $\alpha$. Of the 3 untreated individuals, 1 developed mild disease and the 2 others developed severe CCHF. In contrast, 4 of the 5 ribavirin-treated healthcare workers remained asymptomatic and did not develop measurable anti-CCHFV antibodies, while the fifth suffered only a mild illness. However, another 42 individuals who had been exposed to contaminated blood remained healthy without treatment, making it unclear whether ribavirin therapy actually had an effect.

In 1995, the use of oral ribavirin was reported in 3 severe nosocomial CCHF cases in Pakistan (Fisher-Hoch et al., 1995). All patients became afebrile within $48 \mathrm{~h}$ of the initiation of treatment and recovered completely. In another nosocomial outbreak in Pakistan, ribavirin was used to treat 1 of 2 secondary CCHF cases, who survived (Athar et al., 2003). During another Pakistani CCHF outbreak, 9 patients were treated with oral ribavirin, and 4 survived (Smego et al., 2004). In 2003, in a nosocomial outbreak in a tertiary care hospital in Pakistan, ribavirin was administered to workers involved in the care of the index case (Bangash and Khan, 2003). The secondarily infected patients recovered completely, and none of the 11 health care workers became ill. Finally, it was reported that 6 and 10 CCHF patients in the Golestan Province of Iran and in Istanbul, Turkey, respectively, survived after being treated with ribavirin (Jabbari et al., 2006; Midilli et al., 2007).

4.2.2.2. Historical comparisons. In 2003, Iranian researchers compared case fatality rates among 139 suspected and 69 confirmed CCHF patients, based on oral ribavirin treatment (Mardani et al., 2003). For those with confirmed disease, the survival rate was $69.8 \%$ for treated patients and $41.7 \%$ for untreated cases, while for patients with suspected CCHFV, it was $88.4 \%$ for treated and $54.2 \%$ for untreated cases. The efficacy of oral ribavirin was determined to be $80 \%$ among confirmed and $34 \%$ among suspected CCHF patients.

Ozkurt et al. (2006) compared 22 CCHF patients treated with oral ribavirin to a historical cohort of 38 untreated patients. The case fatality rate was $9.0 \%$ in treated and $10.5 \%$ in the untreated group, a difference that was not statistically significant $(p=0.85)$. The recovery period was shorter in the treated group, but the need for blood products was the same, and the mean hospitalization time and total hospital expenditure did not differ between the groups. However, the groups were not matched for disease severity.

In another study in Iran in 2006, Alavi-Naini et al. evaluated the efficacy of oral ribavirin, and found that 37 (15.7\%) of 236 treated patients and $63.2 \%$ of 19 untreated patients died (Alavi-Naini et al., 2006). The efficacy of treatment was determined to be $75 \%$ and the relative chance of recovery in the treated group was 2.29 times higher than for untreated cases.

Recently, 218 Turkish CCHF patients were retrospectively evaluated for clinical outcome based on oral ribavirin treatment (Elaldi et al., 2009). The case-fatality rate was $9 / 126(7.1 \%)$ in the treated group and 11/92 (11.9\%) in the untreated group. The average interval between disease onset and ribavirin administration was not significantly different among fatal and nonfatal cases in the treated group ( 4.4 days vs. 5.8 days; $p=0.11$ ), and there was no significant difference in the clinical outcome of patients treated within 3 days of disease onset, compared to those treated later $(p=0.14)$. However, this study was criticized for faults in statistical analysis and study design (Ergonul, 2009).

4.2.2.3. Non-randomized clinical trials. In a study of $35 \mathrm{CCHF}$ patients reported from Turkey in 2004, in which 30 patients had severe and 5 mild illness, the overall case-fatality rate was 2.8\% (Ergonul et al., 2004). Oral ribavirin was given to 8 patients with severe disease, all of whom survived, whereas the case-fatality rate was $4.5 \%$ in the 22 patients with severe disease who did not receive the drug.

In 2009, Tasdelen Fisgin et al. evaluated the efficacy of oral ribavirin in Turkish CCHF patients: 21 cases treated within 4 days after the appearance of symptoms and 20 treated beginning 5 days or longer after disease onset (Tasdelen Fisgin et al., 2009). Eleven 
Table 1

Summary of literature published since 1985 on the efficacy of ribavirin therapy of CCHF.

\begin{tabular}{|c|c|c|c|c|}
\hline Country & Treated/total cases & Study type & Prophylaxis or treatment & Reference \\
\hline South Africa & $6 / 9$ & \multirow[t]{7}{*}{ Observational } & Prophylaxis & van de Wal et al. (1985) \\
\hline Pakistan & $3 / 3$ & & Treatment & Fisher-Hoch et al. (1995) \\
\hline Pakistan & $2 / 2$ & & Treatment & Athar et al. (2003) \\
\hline Pakistan & $12 / 12$ & & Prophylaxis & Bangash and Khan (2003) \\
\hline Pakistan & $9 / 9$ & & Treatment & Smego et al. (2004) \\
\hline Iran & $6 / 6$ & & Treatment & Jabbari et al. (2006) \\
\hline Turkey & $10 / 10$ & & Treatment & Midilli et al. (2007) \\
\hline Iran & $61 / 69$ & \multirow{5}{*}{ Historical comparison } & Treatment & Mardani et al. (2003) \\
\hline Iran & $236 / 255$ & & Treatment & Alavi-Naini et al. (2006) \\
\hline Turkey & $22 / 60$ & & Treatment & Ozkurt et al. (2006) \\
\hline Turkey & $126 / 218$ & & Treatment & Elaldi et al. (2009) \\
\hline Turkey & $10 / 50$ & & Treatment & Bodur et al. (2011) \\
\hline Turkey & $8 / 30$ & \multirow[t]{3}{*}{ Non-randomized clinical trial } & Treatment & Ergonul et al. (2004) \\
\hline Turkey & $9 / 25$ & & Treatment & Cevik et al. (2008) \\
\hline Turkey & $41 / 52$ & & Treatment & Tasdelen Fisgin et al. (2009) \\
\hline Iran & $184 / 184$ & \multirow[t]{3}{*}{ Comparison to evaluate timing } & Treatment & Metanat et al. (2006) \\
\hline Iran & $63 / 63$ & & Treatment & Izadi and Salehi (2009) \\
\hline Iran & $155 / 155$ & & Treatment & Sharifi-Mood et al. (2009) \\
\hline Turkey & $64 / 136$ & Randomized clinical trial & Treatment & Koksal et al. (2010) \\
\hline
\end{tabular}

patients were untreated. At days 5-10 after disease onset, the mean platelet counts of the patients who were treated early in their illness were significantly higher than those of patients treated late, and at days 7-9, they were significantly higher than those of the untreated patients. The case fatality rate among early treated patients (5\%) was lower than late treated (10\%) and untreated patients (27\%), but the difference was not statistically significant.

In one of the few studies to evaluate the efficacy of intravenous ribavirin, Cevik and colleagues compared outcomes in 9 severely ill patients, compared to 16 untreated controls (Cevik et al., 2008). They found no statistically significant differences between the 2 groups in terms of case fatality rate, mean duration of hospitalization or the need for blood products, and they accordingly concluded that treatment had no beneficial effect. However, as in the case of the study by Tasdelen Fisgin et al., this report should be interpreted cautiously, as it lacks the statistical power to reach a definite conclusion.

Turkish scientists recently published a retrospective case-control study of the effect of oral ribavirin on viral load and disease progression, which included 10 patients who received the drug for 10 days and 40 who received only supportive therapy (Bodur et al., 2011). There was no significant difference in viral load between the case and control groups at the time of hospital admission. During follow-up, no statistically significant differences were found in the decrease in viral load, reduction in liver enzyme concentrations, increase in platelet count or the case fatality rate. These results suggest that oral ribavirin had no positive effect, but one should note that the number of treated patients was small.

4.2.2.4. Efficacy at different time points. In a case-control study in 2006 in Iran, 84\% of the 89 patients who were treated with oral ribavirin beginning within the first $72 \mathrm{~h}$ of illness onset recovered from the disease, whereas the survival rate of the 95 patients whose treatment began after $72 \mathrm{~h}$ was $74.8 \%$ (Metanat et al., 2006). In another Iranian study, in which 47 of 63 treated patients survived, those treated individuals who survived infection received their initial therapy $24 \mathrm{~h}$ earlier on average than treated patients who died (Izadi, 2009). The interval between the onset of disease or hemorrhage and the initiation of ribavirin administration was the most important variable correlated with survival (Izadi and Salehi, 2009).

In 2009, researchers in the Sistan and Baluchistan Provinces of Iran described a significant difference in recovery among 32 people treated with ribavirin between 2005 and 2007, compared to 123 people treated in 1999-2004 (Sharifi-Mood et al., 2009). All patients from 2005 to 2007 were treated within the first $72 \mathrm{~h}$ of illness onset, whereas only $79 \%$ of those from 1999 to 2004 were treated within that time frame. The case fatality rate among the 2005-2007 cases (3\%) was significantly lower than in the 1999-2004 group (22\%) $(p=0.001)$. Although these data support the early administration of ribavirin, other confounding variables, such as the time of diagnosis and the type of supportive clinical measures should be considered when evaluating the efficacy of therapy.

4.2.2.5. Randomized clinical trials. One hundred thirty-six Turkish CCHF patients were randomized, such that Group A $(n=64)$ received oral ribavirin and supportive therapy, while group $B$ $(n=72)$ received only supportive therapy (Koksal et al., 2010). The two groups were matched for baseline demographic features. There was no statistically significant difference between the two groups in the incubation period, clinical presentation, laboratory findings, time of hospitalization, requirement for platelet infusions, time needed for normalization of platelet counts or survival. Not only did the ribavirin-treated patients not survive longer than the control group, but also their leukocyte counts remained abnormal for a longer period of time.

\subsubsection{Ribavirin therapy: conclusions}

As the variety of conclusions reached by the publications summarized above has shown, the efficacy of ribavirin for the prophylaxis and therapy of CCHF is still an open question. Early anecdotal reports described the recovery of severely ill patients treated with the drug, but more recent studies, including a large randomized clinical trial, have found few or no differences in the course and outcome of illness of treated and untreated patients. Unfortunately, underlying variation in patient populations and the failure of investigators to match cases and controls for disease severity, stage of illness at initiation of treatment and other factors have limited the value of many studies. Because the use of ribavirin is now well established in most countries where CCHF is endemic, there may be ethical objections to performing placebo-controlled trials. Researchers should therefore consider how ribavirin therapy might be further evaluated without violating ethical guidelines. In the meantime, it appears justifiable to continue to administer the 
drug to suspected cases of CCHF in endemic areas until its efficacy has been definitively determined.

\subsection{Specific immunoglobulin}

\subsubsection{Intramuscular anti-CCHF immunoglobulin}

The idea of treating CCHF patients with specific immunoglobulin was first proposed by Chumakov in 1944 (Chumakov, 1945). In 1967, however, Leshchinskaya reported the lack of efficacy of intramuscular injections of convalescent serum in Soviet CCHF patients (Leshchinskaya, 1967). In 1970, the same researchers treated 61 patients with $80 \mathrm{~mL}$ of convalescent serum injected intramuscularly once or twice daily up to day 4 following the onset of hemorrhagic symptoms, and compared them to 88 untreated patients matched in disease severity (Leshchinskaya and Martinenko, 1970). No statistically significant differences in fever duration or lethality were observed, and virus was isolated from the blood even in patients who had received sera for 2 or 3 days. For future studies, the researchers recommended administering convalescent sera by the intravenous route, at earlier time points, for longer duration, in volumes greater than $200 \mathrm{~mL}$ and with higher immunoglobulin titers. Between 1964 and 1968, 98 patients were inoculated with convalescent sera during the first 2-3 days of disease, resulting in a better outcome compared to treatment after the 4th day (Lazarev, 1969).

In 1980, a CCHF patient in a nosocomial outbreak in Dubai who was treated with $300 \mathrm{~mL}$ of convalescent serum survived his illness, and his convalescent period was shorter than that of other survivors (Suleiman et al., 1980). During another nosocomial outbreak in South Africa in 1985, hyperimmune serum was used to treat 5 patients (van Eeden et al., 1985). Two patients received 1 , and 3 patients received 2 injections of $250 \mathrm{~mL}$ of serum intravenously, in addition to supportive therapy. Four patients showed symptomatic improvement for at least $12 \mathrm{~h}$ after the first dose, and again after the second dose. The five patients who received serum all survived. In contrast, two untreated patients who died showed no antibody response at the time of death, suggesting that immune serum therapy could be of decisive importance for survival. The authors suggested that massive and continuous infusion of hyperimmune serum, continuing for $48-72 \mathrm{~h}$, would more effectively influence the disease outcome.

In Bulgaria, specific intramuscular human immunoglobulin (CCHF-Bulin), derived from the plasma of convalescent patients, has been used since 1975 for the post-exposure prophylaxis of persons who have been in contact with suspected CCHF cases. Such individuals receive $3 \mathrm{~mL}$ of immunoglobulin, whereas patients with suspected CCHF receive $6 \mathrm{~mL}$, and confirmed cases receive $6-9 \mathrm{~mL}$ on days $1-5$, or until a therapeutic effect is achieved (Anon., 2008). Case-control studies of the efficacy of immunoglobulin prophylaxis and therapy have not been published.

\subsubsection{Intravenous anti-CCHF immunoglobulin}

Intravenous anti-CCHF immunoglobulin (CCHF-Venin) was tested in 1989 in 7 CCHF patients with severe hemorrhagic manifestations in Bulgaria (Vassilenko et al., 1990). Thirty milliliters were administered together with $30 \mathrm{~mL}$ of CCHF-Bulin and other general supportive measures. All 7 patients recovered quickly, without side effects, and their leukocyte, platelet, and coagulation abnormalities returned to normal. Unfortunately, this study did not include a control group, and as for CCHF-Bulin, data on the efficacy of CCHF-venin based on case-control studies are lacking.

\subsubsection{Conclusions: immunoglobulin therapy}

None of the studies described above have proven the efficacy of specific immunoglobulin for the post-exposure prophylaxis or treatment of $\mathrm{CCHF}$, so these products should be further evalu- ated in well designed clinical trials. To avoid depriving patients of potentially beneficial therapy, one study group might receive ribavirin, while a second group would receive ribavirin plus immune globulin. Such an investigation might be carried out collaboratively between countries that have supplies of CCHF-specific immunoglobulin, such as Bulgaria, and those that experience a higher incidence of disease, such as Turkey or Iran. Alternatively, countries with a higher incidence of CCHF might prepare their own stocks of immune serum and evaluate them as described above.

\subsubsection{Monoclonal antibodies}

Monoclonal antibodies (mAbs) were first used for CCHFV identification in 1987 (Blackburn et al., 1987). Scientists are now attempting to develop anti-CCHFV mAbs for the treatment of patients. mAbs specific to both the Gn and Gc surface glycoproteins were generated to evaluate their neutralization and protection properties; mAbs to Gc, but not Gn, neutralized CCHFV in SW13 cell cultures (Bertolotti-Ciarlet et al., 2005). However, only a subset protected mice after passive immunization, whereas some non-neutralizing mAbs to Gn protected mice from lethal CCHFV challenge. It was concluded that neutralization depends not only on the properties of the antibody, but also on host factors and mechanisms such as antibody-dependent, cell-mediated cytotoxicity.

\section{Summary}

The only available and probably somewhat efficacious CCHF vaccine is an inactivated antigen preparation currently used in Bulgaria. More modern vaccines are under development, but the sporadic nature of the disease even in endemic countries suggests that large trials of vaccine efficacy will be difficult to perform. Finding volunteers may prove challenging, given the growing resistance of populations to vaccines against contagious diseases such as measles or poliomyelitis. The number of people to be vaccinated and the length of time they would have to be followed to confirm protection would have to be carefully defined. Alternatively, many scientists appear to believe that treatment of CCHF with ribavirin is more practical than prevention, but some recently conducted clinical trials appear to counter assumptions of drug efficacy. Immunoglobulin preparations have been used for more than 30 years to prevent and treat CCHF in Bulgaria, but few data have been published, and their efficacy remains unproven. Although recent developments in antibody engineering have raised hopes for novel $\mathrm{mAb}$ therapies, this approach remains in its infancy. Research now relies on two recently developed mouse models for the evaluation of antibodies, antivirals and other forms of prophylaxis and therapy, but the predictive value of testing in these immunodeficient animals has not been defined. Given these many obstacles to progress, CCHF will clearly remain a major challenge to the infectious disease community for the foreseeable future.

\section{Acknowledgment}

We highly appreciate Dr. Mike Bray’s editorial input and support of this paper.

\section{References}

Anon., 2008. ECDC Meeting Report-Consultation on Crimean-Congo haemorrhagic fever prevention and control, Stockholm, Sweden.

Alavi-Naini, R., Moghtaderi, A., Koohpayeh, H.R., Sharifi-Mood, B., Naderi, M., Metanat, M., Izadi, M., 2006. Crimean-Congo hemorrhagic fever in Southeas of Iran. J. Infect. 52, 378-382.

Athar, M.N., Baqai, H.Z., Ahmad, M., Khalid, M.A., Bashir, N., Ahmad, A.M., Balouch, A.H., Bashir, K., 2003. Short report: Crimean-Congo hemorrhagic fever outbreak in Rawalpindi, Pakistan, February 2002. Am. J. Trop. Med. Hyg. 69, 284-287. 
Badalov, M.Y., Butenko, A.M., Karinskaya, G.A., Leshchinskaya, Y.V., Rubin, S.G., Tkachenko, Y.A., Chumakov, M.P., 1969. Serological investigation of the rural population and domestic animals in rostov oblast in connection with the problem of prevention. In: Chumakov, M.P. (Ed.), Arboviruses (Tick-borne and Japanese Encephalitides, Hemorrhagic Fevers, and Other Arboviral Infections). Materials of the 16th Scientific Session of the Institute of Poliomyelitis and Viral Encephalitides. Moscow, USSR [Russian], pp. 117-118.

Bangash, S.A., Khan, E.A., 2003. Treatment and prophylaxis with ribavirin for Crimean-Congo hemorrhagic fever-is it effective? J. Pak. Med. Assoc. 53, 39-41.

Bente, D.A., Alimonti, J.B., Shieh, W.J., Camus, G., Stroher, U.,Zaki, S., Jones, S.M., 2010 Pathogenesis and immune response of Crimean-Congo hemorrhagic fever virus in a STAT-1 knockout mouse model. J. Virol. 84, 11089-11100.

Bereczky, S., Lindegren, G., Karlberg, H., Akerstrom, S., Klingstrom, J., Mirazimi, A 2010. Crimean-Congo hemorrhagic fever virus infection is lethal for adult type I interferon receptor-knockout mice. J. Gen. Virol. 91, 1473-1477.

Bertolotti-Ciarlet, A., Smith, J., Strecker, K., Paragas, J., Altamura, L.A., McFalls, J.M. Frias-Staheli, N., Garcia-Sastre, A., Schmaljohn, C.S., Doms, R.W., 2005. Cellula localization and antigenic characterization of Crimean-Congo hemorrhagic fever virus glycoproteins. J. Virol. 79, 6152-6161.

Blackburn, N.K., Besselaar, T.G., Shepherd, A.J., Swanepoel, R., 1987. Preparation and use of monoclonal antibodies for identifying Crimean-Congo hemorrhagic feve virus. Am. J. Trop. Med. Hyg. 37, 392-397.

Bodur, H., Erbay, A., Akıncı, E., Öngürü, P., Bayazıt, N., Eren, S.S., Kubar, A., 2011. Effect of oral ribavirin treatment on the viral load and disease progression in Crimean-Congo hemorrhagic fever. Int. J. Infect. Dis. 15, e44-e47.

Borio, L., Inglesby, T., Peters, C.J., Schmaljohn, A.L., Hughes, J.M., Jahrling, P.B., Ksiazek T., Johnson, K.M., Meyerhoff, A., O’Toole, T., Ascher, M.S., Bartlett, J., Breman, J.G. Eitzen Jr., E.M., Hamburg, M., Hauer, J., Henderson, D.A., Johnson, R.T., Kwik, G. Layton, M., Lillibridge, S., Nabel, G.J., Osterholm, M.T., Perl, T.M., Russell, P., Tonat, K., 2002. Hemorrhagic fever viruses as biological weapons: medical and public health management. J. Am. Med. Assoc. 287, 2391-2405.

Brehm, M.A., Shultz, L.D., Greiner, D.L., 2010. Humanized mouse models to study human diseases. Curr. Opin. Endocrinol. Diabetes Obes. 17, 120-125.

Bronze, M.S., Huycke, M.M., Machado, L.J., Voskuhl, G.W., Greenfield, R.A., 2002. Viral agents as biological weapons and agents of bioterrorism. Am. J. Med. Sci. 323 $316-325$.

Carter, S., 2009. Rare virus poses new threat to troops. The Washington Times, Washington, DC. http://www.washingtontimes.com/news/2009/nov/06/rarevirus-poses-new-threat-to-troops.

Casals, J., 1969. Antigenic similarity between the virus causing Crimean hemorrhagic fever and Congo virus. Proc. Soc. Exp. Biol. Med. 131, 233-236.

Cevik, M.A., Elaldi, N., Akinci, E., Onguru, P., Erbay, A., Buzgan, T., Uzun, R., Kubar, A., Bodur, H., 2008. A preliminary study to evaluate the effect of intravenous ribavirin treatment on survival rates in Crimean-Congo hemorrhagic fever. J. Infect. 57, 350-351.

Christova, I., Kovacheva, O., Georgieva, G., Ivanova, S., Argirov, D., 2010. Vaccine against Congo-Crimean haemorhagic fever virus-Bulgarian input in fighting the disease. Probl. Infect. Parasit. Dis. 37, 7-8.

Chumakov, M.P., 1945. A new tick-borne virus disease-Crimean hemorrhagic fever (acute infectious capillary toxicosis). In: Sokolov, A.E., Chumakov, M.P., Kolachev, A.A. (Eds.), Crimean Hemorrhagic Fever (Acute Infectious Capillary Toxicosis). Izdanie Otdel'noj Primorskoj Armii, Simferopol. USSR [Russian], pp. $13-43$

Elaldi, N., Bodur, H., Ascioglu, S., Celikbas, A., Ozkurt, Z., Vahaboglu, H., Leblebicioglu, H., Yilmaz, N., Engin, A., Sencan, M., Aydin, K., Dokmetas, I., Cevik, M.A., Dokuzoguz, B., Tasyaran, M.A., Ozturk, R., Bakir, M., Uzun, R., 2009. Efficacy of oral ribavirin treatment in Crimean-Congo haemorrhagic fever: a quasiexperimental study from Turkey. J. Infect. 58, 238-244.

Ergonul, O., 2006. Crimean-Congo haemorrhagic fever. Lancet Infect Dis. 6, 203-214.

Ergonul, O., 2007. Clinical and pathologic features of Crimean-Congo hemorrhagic fever. In: Ergonul, O., Whitehouse, C.A. (Eds.), Crimean-Congo Hemorrhagic Fever-A Global Perspective. Springer, Vienna, Austria, pp. 207-220.

Ergonul, O., 2009. Biases and misinterpretation in the assessment of the efficacy of oral ribavirin in the treatment of Crimeane-Congo hemorrhagic fever. J. Infect. 59, 284-301.

Ergonul, O., Celikbas, A., Dokuzoguz, B., Eren, S., Baykam, N., Esener, H., 2004 Characteristics of patients with Crimean-Congo hemorrhagic fever in a recent outbreak in Turkey and impact of oral ribavirin therapy. Clin. Infect. Dis. 39, 284-287.

Ergonul, O., Tuncbilek, S., Baykam, N., Celikbas, A., Dokuzoguz, B., 2006. Evaluation of serum levels of interleukin (IL)-6 IL-10, and tumor necrosis factor-alpha in patients with Crimean-Congo hemorrhagic fever. J. Infect. Dis. 193, 941-944.

Ergonul, O., Whitehouse, C.A., 2007. Crimean-Congo Hemorrhagic Fever-A Global Perspective. Springer, Vienna, Austria.

Fagbami, A.H., Tomori, O., Fabiyi, A., Isoun, T.T., 1975. Experimantal Congo virus (Ib-AN 7620) infection in primates. Virologie 26, 33-37.

Fisher-Hoch, S.P., Khan, J.A., Rehman, S., Mirza, S., Khurshid, M., McCormick, J.B., 1995. Crimean Congo-haemorrhagic fever treated with oral ribavirin. Lancet $346,472-475$.

Graci, J.D., Cameron, C.E., 2006. Mechanisms of action of ribavirin against distinct viruses. Rev. Med. Virol. 16, 37-48.

Haenni, A.-L., de Miranda, J.R., Falk, B.W., Goldbach, R., Mayo, M.A., Shirako, Y., Toriyama, S., 2005. Family Bunyaviridae. In: Fauquet, C.M., Mayo, M.A., Maniloff, J., Desselberger, U., Ball, L.A. (Eds.), Virus Taxonomy-Eighth Report of the International Committee on Taxonomy of Viruses. Elsevier/Academic Press, San Diego, USA, pp. 695-723.
Hemachudha, T., Griffin, D.E., Giffels, J.J., Johnson, R.T., Moser, A.B., Phanuphak, P., 1987. Myelin basic protein as an encephalitogen in encephalomyelitis and polyneuritis following rabies vaccination. N. Engl. J. Med. 316, 369-374.

Izadi, S., Salehi, M., 2009. Evaluation of the efficacy of ribavirin therapy on survival of Crimean-Congo hemorrhagic fever patients: a case-control study. Jpn. J. Infect. Dis. 62, 11-15.

Izadi, S.S.M., 2009. Evaluation of the efficacy of ribavirin therapy on survival of Crimean-Congo hemorrhagic fever patients: a case-control study. Jpn. J. Infect. Dis., 11-15.

Jabbari, A., Besharat, S., Abbasi, A., Moradi, A., Kalavi, K., 2006. Crimean-Congo hemorrhagic fever: case series from a medical center in Golestan province, Northeast of Iran (2004). Indian J. Med. Sci. 60, 327-329.

Jelinek, T., 2008. Japanese encephalitis vaccine in travelers. Expert Rev. Vaccines 7, 689-693.

Joubert, J.R., King, J.B., Rossouw, D.J., Cooper, R., 1985. A nosocomial outbreak of Crimean-Congo haemorrhagic fever at Tygerberg Hospital. Part III. Clinical pathology and pathogenesis. S. Afr. Med. J. 68, 722-728.

Koksal, I., Yilmaz, G., Aksoy, F., Aydin, H., Yavuz, I., Iskender, S., Akcay, K., Erensoy, S. Caylan, R., Aydin, K., 2010. The efficacy of ribavirin in the treatment of CrimeanCongo hemorrhagic fever in Eastern Black Sea region in Turkey. J. Clin. Virol. 47, 65-68.

Lazarev, V.N., 1969. Treatment of Crimean hemorrhagic fever patients with convalescent sera. In: Chumakov, M.P. (Ed.), Arboviruses (Tick-borne and Japanese Encephalitides, Hemorrhagic Fevers, and Other Arboviral Infections). Materials of the 16th Scientific Session of the Institute of Poliomyelitis and Vira Encephalitides, vol. 2. Academy of Medical Sciences, Moscow, USSR [Russian] pp. 142-143.

Leshchinskaya, Y.V., 1967. Crimean Hemorrhagic Fever Clinical Picture and Comparison with Other Hemorrhagic Fever Types. Dissertation. USSR Academy of Medical Science of USSR, Moscow, USSR.

Leshchinskaya, Y.V., Martinenko, I.N., 1970. Certain problems in Crimean hemorrhagic fever therapy. In: Chumakov, M.P. (Ed.), Crimean Hemorrhagic Fever-Materials of the 3rd Scientific-practical Conference in Rostov-na-Donu Region. Rostov-na-Donu, USSR [Russian], pp. 111-115.

Mardani, M., Jahromi, M.K., Naieni, K.H., Zeinali, M., 2003. The efficacy of oral ribavirin in the treatment of crimean-congo hemorrhagic fever in Iran. Clin. Infect. Dis. 36, 1613-1618.

Mardani, M., Keshtkar-Jahromi, M., 2007. Crimean-Congo hemorrhagic fever. Arch. Iran Med. 10, 204-214.

Metanat, M., Sharifi-Mood, B., Salehi, M., Alavi-Naini, R., 2006. Clinical outcomes in Crimean-Congo hemorrhagic fever: a five-years experience in the treatment of patients in oral ribavirin. Int. J. Virol. 2, 21-24.

Midilli, K., Gargili, A., Ergonul, O., Sengoz, G., Ozturk, R., Bakar, M., Jongejan, F., 2007. Imported Crimean-Congo hemorrhagic fever cases in Istanbul. BMC Infect. Dis. 7, 54

Ozkurt, Z., Kiki, I., Erol, S., Erdem, F., Yilmaz, N., Parlak, M., Gundogdu, M., Tasyaran, M.A., 2006. Crimean-Congo hemorrhagic fever in Eastern Turkey: clinical features, risk factors and efficacy of ribavirin therapy. J. Infect. 52, 207-215.

Papa, A., Bino, S., Velo, E., Harxhi, A., Kota, M., Antoniadis, A., 2006 Cytokine levels in Crimean-Congo hemorrhagic fever. J. Clin. Virol. 36, 272-276.

Paragas, J., Whitehouse, C.A., Endy, T.P., Bray, M., 2004. A simple assay for determining antiviral activity against Crimean-Congo hemorrhagic fever virus. Antiviral Res. 62, 21-25.

Sharifi-Mood, B., Mardani, M., Keshtkar-Jahromi, M., Rahnavardi, M., Hatami, H. Metanat, M., 2008. Clinical and epidemiologic features of Crimean-Congo hemorrhagic fever among children and adolescents from southeastern Iran. Pediatr. Infect. Dis. J. 27, 561-563.

Sharifi-Mood, B., Metanat, M., Ghorbani-Vaghei, A., Fayyaz-Jahani, F., Akrami, E., 2009. The outcome of patients with Crimean-Congo hemorrhagic Fever in zahedan, southeast of iran: a comparative study. Arch. Iran Med. 12 151-153.

Shepherd, A.J., Leman, P.A., Swanepoel, R., 1989. Viremia and antibody response of small African and laboratory animals to Crimean-Congo hemorrhagic fever virus infection. Am. J. Trop. Med. Hyg. 40, 541-547.

Simpson, D.I., Knight, E.M., Courtois, G., Williams, M.C., Weinbren, M.P., Kibukamusoke, J.W., 1967. Congo virus: a hitherto undescribed virus occurring in Africa I. Human isolations-clinical notes. East Afr. Med. J. 44, 86-92.

Simpson, D.I.H., Williams, M.C., Woodal, I.P., 1965. Four cases of human infection with the Congo agent. East Afr. Virus Res. Inst. Rep., 27-28.

Smego Jr., R.A., Sarwari, A.R., Siddiqui, A.R., 2004. Crimean-Congo hemorrhagic fever: prevention and control limitations in a resource-poor country. Clin. Infect. Dis. $38,1731-1735$.

Smirnova, S.E., 1979. A comparative study of the Crimean hemorrhagic fever-Congo group of viruses. Arch. Virol. 62, 137-143.

Smirnova, S.E., Zubri, G.L., Savinov, A.P., Chumakov, M.P., 1973. Pathogenesis of experimental Crimean hemorrhagic fever infection in newborn white mice. Acta Virol. 17, 409-415.

Spik, K., Shurtleff, A., McElroy, A.K., Guttieri, M.C., Hooper, J.W., SchmalJohn, C., 2006 Immunogenicity of combination DNA vaccines for Rift Valley fever virus, tickborne encephalitis virus, Hantaan virus, and Crimean Congo hemorrhagic fever virus. Vaccine 24, 4657-4666.

Suleiman, M.N., Muscat-Baron, J.M., Harries, J.R., Satti, A.G., Platt, G.S., Bowen, E.T. Simpson, D.I., 1980. Congo/Crimean haemorrhagic fever in Dubai: an outbreak at the Rashid Hospital. Lancet 2, 939-941. 
Swanepoel, R., Gill, D.E., Shepherd, A.J., Leman, P.A., Mynhardt, J.H., Harvey, S., 1989. The clinical pathology of Crimean-Congo hemorrhagic fever. Rev. Infect. Dis. 11 (suppl. 4), S794-S800.

Tasdelen Fisgin, N., Ergonul, O., Doganci, L., Tulek, N., 2009. The role of ribavirin in the therapy of Crimean-Congo hemorrhagic fever: early use is promising. Eur. J. Clin. Microbiol. Infect. Dis. 28, 929-933.

Tezer, H., Sucakli, I.A., Sayli, T.R., Celikel, E., Yakut, I., Kara, A., Tunc, B., Ergonul, O. 2010. Crimean-Congo hemorrhagic fever in children. J. Clin. Virol. 48, 184-186.

Tignor, G.H., Hanham, C.A., 1993. Ribavirin efficacy in an in vivo model of Crimean-Congo hemorrhagic fever virus (CCHF) infection. Antiviral Res. 22, 309-325.

Tkachenko, Y.A., Butenko, A.M., Badalov, M.Y., Zaradova, T.I., Chumakov, M.P., 1971. Investigation of immunogenic activity of killed brain vaccine against Crimean hemorrhagic fever viral hemorrhagic fevers. In: Chumakov, M.P. (Ed.), Viral Hemorrhagic Fevers-Crimean Hemorrhagic Fever, Omsk Hemorrhagic Fever, Hemorrhagic Fever with Renal Syndrom. Trudy Instituta Poliomielita i Virusnykh Entsefalitov. vol. XIX. USSR Academy of Medical Sciences, Moscow, USSR [Russian], pp. 119-129.

Tkachenko, Y.A., Butenko, A.M., Butenko, S.A., Zavodova, T.I., Chumakov, M.P., 1970. Prophylactic characteristics of a protective Crimean hemorrhagic fever vaccine. In: Chumakov, M.P. (Ed.), Crimean Hemorrhagic Fever-Materials of the 3rd Scientific-practical Conference in Rostov-na-Donu Region, Rostov-na-Donu. USSR [Russian], pp. 136-138. van de Wal, B.W., Joubert, J.R., van Eeden, P.J., King, J.B., 1985. A nosocomial outbreak of Crimean-Congo haemorrhagic fever at Tygerberg Hospital. Part IV. Preventive and prophylactic measures. S. Afr. Med. J. 68, 729-732.

van Eeden, P.J., van Eeden, S.F., Joubert, J.R., King J.B., van de Wal, W., Michell, W.L. 1985. A nosocomial outbreak of Crimean-Congo hemorrhagic fever at Tygerberg hospital. Part II. Management of patients. S. Afr. Med. J. 68, 718-721.

Vasilenko, S.M., 1973. Results of the investigation on etiology, epidemiology features and the specific prophylaxis of Crimean hemorrhagic fever (CHF) in Bulgaria. In: Papaevangelou, G.J. (Ed.), Ninth International Congress on Tropical Medicine and Malaria. October 14-21, Athens, Greece, pp. 32-33.

Vassilenko, S.M., Vassilev, T.L., Bozadjiev, L.G., Bineva, I.L., Kazarov, G.Z., 1990. Specific intravenous immunoglobulin for Crimean-Congo haemorrhagic fever. Lancet 335, 791-792.

Vorou, R., Pierroutsakos, I.N., Maltezou, H.C., 2007. Crimean-Congo hemorrhagic fever. Curr. Opin. Infect. Dis. 20, 495-500.

Watts, D.M., Ussery, M.A., Nash, D., Peters, C.J., 1989. Inhibition of Crimean-Congo hemorrhagic fever viral infectivity yields in vitro by ribavirin. Am. J. Trop. Med. Hyg. 41, 581-585.

Woodall, J.P., 2007. Personal reflections. In: Ergonul, O., Whitehouse, C.A. (Eds.) Crimean-Congo Hemorrhagic Fever-A Global Perspective. Springer, Vienna, Austria, pp. 23-32.

Yilmaz, R., Kundak, A.A., Ozer, S., Esmeray, H., 2009. Successful treatment of severe Crimean-Congo hemorrhagic fever with supportive measures without ribavirin and hypothermia. J. Clin. Virol. 44, 181-182. 